\title{
Blood Flow Dynamics After Laser Therapy of Port Wine Stain Birthmarks
}

\author{
Yu-Chih Huang, MS ${ }^{1,2}$, Nadia Tran, BA ${ }^{1}$, Peter R. Shumaker, MD $^{3}$, Kristen Kelly, MD ${ }^{1,4}$, E. \\ Victor Ross, MD ${ }^{3}$, J. Stuart Nelson, MD, PhD ${ }^{1,4,5}$, and Bernard Choi, PhD ${ }^{1,5}{ }^{*}$ \\ ${ }^{1}$ Beckman Laser Institute and Medical Clinic, University of California, Irvine, California 92612 \\ 2 Department of Electrical Engineering and Computer Science, University of California, Irvine, \\ California 92697 \\ ${ }^{3}$ Laser and Cosmetic Dermatology Center, Scripps Clinic, San Diego, California 92130 \\ 4 Department of Dermatology, University of California, Irvine, California 92697 \\ ${ }^{5}$ Department of Biomedical Engineering, University of California, Irvine, California 92697
}

\begin{abstract}
Background and Objective-During laser therapy of port wine stain (PWS) birthmarks, regions of perfusion may persist. We hypothesize that such regions are not readily observable even when laser surgery is performed by highly experienced clinicians. The objective of this study was to use objective feedback to assess the acute vascular response to laser therapy.
\end{abstract}

Study Design/Materials and Methods-A clinic-friendly laser speckle imaging (LSI) instrument was developed to provide the clinician with real-time images of blood flow during laser therapy. Images were acquired from patients undergoing laser therapy of PWS birthmarks at Scripps Clinic and the Beckman Laser Institute and Medical Clinic. Blood flow maps were extracted from the acquired imaging data. Histogram-based analysis was applied in grading the degree of heterogeneity present in the blood flow maps after laser therapy.

Results-Collectively, two types of patient responses were observed in response to laser exposure: (1) an immediate increase in perfusion within minutes after laser therapy; and (2) an overall decrease in blood perfusion $\sim 1$ hour after laser therapy, with distinct regions of persistent perfusion apparent in the majority of post-treatment blood-flow images. A comparison of blood flow in PWS and adjacent normal skin demonstrated that PWS blood flow can be greater than, or sometimes equivalent to, that of normal skin.

Conclusion-In general, a decrease in skin perfusion is observed during pulsed laser therapy of PWS birthmarks. However, a heterogeneous perfusion map was frequently observed. These regions of persistent perfusion may be due to incomplete photocoagulation of the targeted vessels. We hypothesize that immediate retreatment of these regions identified with LSI, will result in enhanced removal of the PWS vasculature.

\section{Keywords}

laser Doppler perfusion imaging; laser speckle contrast analysis (LASCA); laser Doppler flowmetry; non-invasive blood flow imaging; laser therapy; vascular birthmarks

*Correspondence to: Bernard Choi, PhD, Beckman Laser Institute and Medical Clinic, University of California, 1002 Health Sciences Road East, Irvine, CA 92612. choib@uci.edu. 


\section{INTRODUCTION}

Port wine stain (PWS) birthmarks are vascular malformations consisting of ectatic blood vessels in the papillary and upper reticular dermis [1]. Pulsed dye laser (PDL) therapy is the standard approach to treat these birthmarks. Unfortunately, complete disappearance of the PWS infrequently occurs, with a success rate of $\sim 10 \%[2,3]$. Previous studies have presented results suggesting that specific PWS skin characteristics are associated with treatment outcome, yet the outcome largely remains unpredictable on an individual patient basis and even from site to site on the same patient [4].

The intended goal of laser therapy is acute photocoagulation of the PWS microvasculature. A common metric used clinically to assess photocoagulation is purpura (i.e., bluish-gray skin discoloration) formation. However, purpura formation may indicate other phenomena besides vessel photocoagulation. For example, laser-induced rupture of blood vessels can lead to increased hemoglobin content diffusing into the perivascular space, without concurrent vessel photocoagulation [5]. Such an event also would manifest itself as purpura formation. Moreover, due to the overall poor success rate of laser therapy to achieve complete PWS removal, a reasonable conclusion is that purpura formation itself is insufficient as a prognostic indicator. Thus, there is a critical need for an alternate method to assess the degree of photocoagulation achieved during therapy.

To assess blood flow in PWS vessels, only a few studies exist in the peer-reviewed literature. In general, either laser Doppler imaging [6-8] or Doppler optical coherence tomography [911] has been employed. In particular, with Doppler optical coherence tomography evaluation of PWS skin, the authors acknowledge the potential of blood flow characterization as a means to monitor PWS skin during laser therapy and retreat if photocoagulation of targeted vessels has not occurred.

Laser Doppler flowmetry (LDF), a noninvasive method to characterize tissue perfusion, has been commercially available since the early 1980s. However, LDF is essentially a point measurement instrument, and hence can characterize only a small fraction of the PWS vasculature. Laser Doppler perfusion imaging (LDPI) employs a mechanical scanner to achieve a two-dimensional map of tissue perfusion. Moreover, this instrument requires a long scan time, even for modest (i.e., 256×256) pixel dimensions [6], which increases the likelihood of image corruption by motion artifacts. Thus, to assess the degree of photocoagulation in a practical manner, there is critical need for a faster vascular imaging platform.

To elucidate the blood flow dynamics during laser surgery, a clinical laser speckle imaging (LSI) instrument has been developed [12], which can provide wide field images to objectively characterize PWS skin perfusion dynamics in real time at specific sites on the lesion. LSI relies on acquisition and analysis of a single image captured at an exposure time that is considerably longer than a characteristic correlation time associated with the fluctuation frequency [13]. Under such conditions, motion of particles, such as red blood cells, induces a blurring of the speckle pattern and hence a loss in speckle contrast [14-16]. A simplified speckle imaging equation has been derived which relates speckle contrast with local blood flow information $[12,13]$. The advantages of LSI over laser Doppler imaging and Doppler optical coherence tomography include speed, cost, simplicity, and potential ease of integration into current laser systems.

In the present study, a clinical LSI instrument was used to collect speckle reflectance images from PWS patients undergoing PDL therapy, with two specific aims: (1) determine the mean perfusion change associated with PDL therapy; and (2) determine the frequency at which regions of persistent perfusion are present. 


\section{MATERIALS AND METHODS}

\section{Clinical LSI Instrument}

We have used LSI to measure the blood perfusion of PWS skin [12]. A primary design consideration was to collect raw speckle images with subjects positioned as comfortably as possible. To this end, an articulated arm was used as the base platform to provide flexibility in instrument positioning. Continuous-wave HeNe laser light $(\lambda=633 \mathrm{~nm}, 30 \mathrm{~mW}$, Edmund Industrial Optics, Barrington, NJ) is delivered to the target area with an optical fiber and diffusion glass (Fig. 1). We selected this method for beam expansion over a diverging lens because of the higher degree of homogeneity within the irradiated field that could be achieved at a comparable speckle contrast (unpublished data). Raw speckle reflectance images are collected with a 12-bit, thermoelectrically cooled CCD camera (Retiga2000R, QImaging, Burnaby, BC, Canada) with a sensor size of $1,600 \times 1,200(\mathrm{H} \times \mathrm{V})$ pixels. Image data are transferred via FireWire connection to a PC for storage, processing, and visualization. Customwritten LabVIEW software (Version 8.0, National Instruments, Austin, TX) is used to control all aspects of image acquisition and processing. With a macro lens (Edmund Industrial Optics) attached to the camera, the field of view has dimensions of $4 \mathrm{~cm} \times 3 \mathrm{~cm}$.

\section{Human Subjects}

LSI measurements were performed at two research sites: the Beckman Laser Institute and Medical Clinic, University of California, Irvine; and the Laser and Cosmetic Dermatology Center, Scripps Clinic, San Diego, CA. A total of 22 subjects (13 female, 9 male) with facial PWS enrolled in the study. The mean age of the subject pool was 29.8 years (age 10-59 years). Seventy-six measurement sessions were evaluated. The measurement protocol was approved by the Institutional Review Boards at University of California, Irvine and Scripps Clinic. Prior to imaging, each subject reviewed and signed an informed consent form.

\section{Imaging Protocol}

LSI before, and 40minutes after, laser treatment-Speckle image data were collected from 20 subjects, both before, and $\sim 40$ minutes after, laser therapy. Various lasers were used to treat these subjects, including the modified V-beam $(577 \mathrm{~nm}$, Candela Laser Corporation, Wayland, MA), V-beam (Perfecta, $595 \mathrm{~nm}$, Candela Laser Corporation), C-beam (585 nm, Candela Laser Corporation), Cynergy (595 nm and 1,064 nm, Cynosure, Inc., Westford, MA), and alexandrite (755 nm, Candela Laser Corporation) (see Table A1 for complete list of treatment parameters). For all treatments, laser therapy was performed in conjunction with cryogen spray cooling (CSC). After treatment, patients undergoing general anesthesia were allowed to recover, after which a second set of speckle images were collected.

LSI for monitoring dynamic blood perfusion-To study blood perfusion dynamics immediately after laser therapy, speckle images were collected from two subjects before and at intervals of 5 minutes, for 30 minutes after laser therapy.

\section{Data Analysis}

To quantify changes in blood perfusion, the mean speckle flow index (SFI) value of the treated area was calculated using a simplified speckle imaging algorithm [17]. Percentage mean changes in mean SFI value were calculated via Equation (1):

$$
\Delta \mathrm{SFI}_{\text {mean }} \%=\frac{\left(\mathrm{SFI}_{\text {after,mean }}-\mathrm{SFI}_{\text {before,mean }}\right)}{\mathrm{SFI}_{\text {before, mean }}} \times 100 \%
$$


To quantify the heterogeneity in the resultant blood perfusion map collected, histogram-based analysis was performed on post-treatment images. We arbitrarily selected post-treatment data in which at least a $20 \%$ reduction in perfusion was observed, to focus attention on data sets in which a clear reduction in mean perfusion was observed. The interquartile range (IQR) was applied to estimate the range in the SFI values within the treated area in each SFI image. IQR is the distance between the 75th percentile and the 25th percentile. Therefore, the IQR is minimally affected by outliers or extreme values. We then calculated the degree of heterogeneity $(\mathrm{DoH})$ as:

$$
\text { Degree of heterogeneity }(\mathrm{DoH})=\frac{\mathrm{IQR}}{\mathrm{SFI}_{\mathrm{after} \text { mean }}}
$$

To facilitate analysis, the DoH results were separated into four groups: 0.1-0.3 (i.e., low DoH), $0.3-0.5$ (moderate), $0.5-0.7$ (high), and 0.7-0.9 (very high).

\section{RESULTS}

\section{Purpura Formation Is Insufficient as a Prognostic Indicator of Photocoagulation}

To demonstrate this point, we present a representative example in which regions of persistent perfusion are visible in the region of visible purpura, immediately after laser treatment (Fig. 2).

\section{Summary of Mean Perfusion Reduction Assessment}

Collectively, we observed a mean perfusion reduction of at least $20 \%$, in $71 \%(54 / 76)$ of the treatment sessions (Fig. 3). In Figures 4 and 5, we present two representative cases of perfusion reduction with either moderate or low $\mathrm{DoH}$, respectively, in the resultant perfusion image. Note that the images in Figures 2 and 4 were acquired from the same patient. However, images in Figure 4 were acquired during a second treatment session which occurred 20 weeks later.

\section{Summary of DoH Assessment}

As described above, we quantified DoH for the 54 measurement sessions in which a mean perfusion reduction of $20 \%$ or greater was achieved (Fig. 6). Of these sessions, 21 (38\%) were graded as low DoH, 21 (38\%) as moderate DoH, $12(22 \%)$ as high DoH, and $1(2 \%)$ as very high DoH. These data demonstrate that a heterogeneous perfusion field (i.e., high and very high DoH) occurs frequently ( $24 \%$ of the 54 treatment sessions), suggesting the potential role of LSI for image guidance during laser therapy of PWS birthmarks.

\section{Dynamics of Blood Perfusion Immediately Following Laser Therapy}

Based on preliminary observations that blood perfusion immediately after laser therapy tends to be higher relative to baseline conditions. Hence, we studied the course of blood perfusion changes during the 30 minutes period immediately following laser therapy. We calculated mean SFI values from a ROI in SFI images before(baseline) and 5, 10, 20, and 30 minutes after laser therapy (Fig. 7). Compared to the baseline image, perfusion was elevated from baseline values by $29 \%$ at 5 minutes after laser treatment, $12 \%$ at 10 minutes, and $11 \%$ at 20 minutes following treatment. A similar time course of perfusion dynamics was observed for both subjects. These preliminary data suggest that a period of $\sim 30$ minutes after treatment is required for the overall acute effects of laser therapy to be adequately assessed properly. 


\section{DISCUSSION}

In a previous study [15], we presented data demonstrating that LSI has a linear response range for flow rates of 0 to $5 \mathrm{~mm} / \mathrm{seconds}$, with a velocity dynamic range of $60 \mathrm{~dB}$. Due to the lowflow nature of PWS vessels, this range is expected to cover the flow rate range of PWS vessels. The corresponding SFI value range is from 0 to $\sim 1,000$. For experiments in which we observed a $>20 \%$ reduction in perfusion, we compute mean SFI values of $\sim 1,100$ and $\sim 700$ from images taken before and after treatment, respectively.

The formation of purpura after PDL therapy is associated with inflammation, edema, and hypoxia in the upper layers of the skin. The concomitant change in color has been associated with photocoagulation of the vasculature and hence is routinely used as a clinical endpoint of PDL therapy $[18,19]$. In this study, purpura was used as a treatment endpoint, yet our SFI data indicate that an appreciable decrease in blood perfusion was observed only $71 \%$ of the time (Fig. 3). Of these "successful cases," regions of persistent perfusion (i.e., either high or very high DoH) occurred $24 \%$ of the time (Fig. 6). These data strongly suggest that an alternate treatment endpoint is required, as purpura formation alone appears to be a suboptimal predictive factor of photocoagulation. With use of clinical LSI, the location of these regions could be studied and immediately retreated during the same patient visit. We expect that such a treatment protocol would lead to a significant improvement in treatment outcome, and we believe further study is warranted.

For experiments in which we observed a $>20 \%$ reduction in perfusion, we compute mean SFI values of $\sim 1,100$ and $\sim 700$ from images taken before and after treatment, respectively. The range of pretreatment SFI values was between 500 and 3,000. To estimate the precision of our instrument, we conducted experiments in which we collected speckle data from a skinsimulating phantom. The phantom contained a well filled with Intralipid to simulate blood perfusion. From 10 such experiments, we computed a mean SFI value of $819 \pm 24$, and a coefficient of variation of $3 \%$. Hence, the mean change in SFI values associated with laser treatment of PWS birthmarks, is an order of magnitude greater than the precision of our instrument, and these data collectively suggest that our DoH metric is appropriate to characterize quantitatively the treatment response.

We postulate that the presence of regions of persistent perfusion indicates an incomplete treatment outcome. Active blood flow through the undertreated, patent microvasculature of the PWS can serve as seed locations from which new PWS vessels can sprout, resulting in at least partial reformation of an abnormal vascular network. Hence, immediate retreatment of the regions of persistent perfusion is expected to minimize the chance that PWS vessels will revascularize.

In this study, we used LSI as a passive perfusion monitoring tool and hence did not use LSI to dictate which laser systems or parameters were employed by the treating clinicians. The wide range of treatment parameters used in this study (Table A1) highlights the need to establish an objective method to determine which treatment parameters to utilize on each patient. Several published studies [20-22] have shown that the treatment efficacy may have a strong dependence on individual PWS skin characteristics, such as vessel size, diameter, and depth. Hence, observation of regions of persistent perfusion may suggest that an alternate treatment protocol would target more effectively an individual patient's vasculature [23]. With use of clinical LSI, different treatment parameters (i.e., pulse duration, spot size, wavelength, etc.) could be readily evaluated to ascertain which combination yields the greatest degree of vascular shutdown.

Immediately after laser therapy, we typically observe an increase in perfusion (Fig. 7A,B), which was an unexpected finding. We believe that the cause of this immediate increase is the 
hyperemic response of vasculature which remains uncoagulated after pulsed laser irradiation. Heat diffusion from the excited hemoglobin molecules homogenizes the elevated temperature field. To remove efficiently the excess heat, the blood flow in patent vasculature increases, resulting in a measurable increase in SFI values. Our preliminary data collected from two subjects suggest that steady-state perfusion is reached $\sim 30$ minutes after laser therapy (Fig. 7). Hence, the data we present in Figures 2-5 represent the steady-state response of PWS vasculature to pulsed laser therapy.

Based on Hohenleutner et al. [24], photocoagulation of the vessel wall with the PDL, is generally limited to a maximum depth of $0.65 \mathrm{~mm}$, even in areas at which deep vessels are not shielded by more superficial vessels. The authors' data suggested a mean coagulation depth of $0.37 \mathrm{~mm}$. In vitro experiments in our lab on tissue phantoms suggest that our LSI instrument possesses a contrast-to-noise ratio greater than unity for depths $<0.6 \mathrm{~mm}$ (unpublished data). Collectively, these data suggest that the skin depth interrogated with our LSI instrument matches well with the depth of effect of the PDL. Furthermore, they suggest that the regions of persistent perfusion commonly observed correspond to insufficiently coagulated, superficial vasculature.

After treatment, most subjects were asked to apply an ice pack to the treated skin. Hence, the treated skin was actively cooled between the pre- and post-treatment imaging sessions.

Preliminary data collected from untreated normal skin of volunteers suggest that ice-pack application induces a slight increase $(16 \%, n=3)$ in SFI values, followed by a return to nearbaseline values within $\sim 3$ minutes. We attribute the increase in SFI due to thermoregulation of the skin. Thus, our preliminary data suggest that the observed perfusion reduction after laser therapy is not due to an artifact of active cooling with an ice pack.

\section{CONCLUSIONS}

In general, a decrease in skin perfusion is observed during pulsed laser therapy of PWS birthmarks. However, a heterogeneous perfusion map was frequently observed. These regions of persistent perfusion may be due to incomplete photocoagulation of the targeted vessels. Our preliminary data suggest that $\sim 30$ minutes is required to differentiate regions of persistent perfusion from vasculature which temporarily dilates to remove excess heat. We hypothesize that immediate retreatment of these regions identified with LSI, will result in enhanced removal of the PWS vasculature. Future study is warranted.

\section{Acknowledgments}

This work was supported in part by grants obtained from the Arnold and Mabel Beckman Foundation, the A. Ward Ford Foundation (BC), the National Institutes of Health (AR51443 to K.M.K. and AR47551, AR248458 and EB002495 to J.S.N.), and the National Institutes of Health Laser Microbeam and Medical Program (LAMMP, a NIH Biomedical Technology Resource, grant no. P41-RR01192, at University of California, Irvine). The Authors would like to acknowledge the contributions of PhD student Austin Moy.

Contract grant sponsor: Arnold and Mabel Beckman Foundation; Contract grant sponsor: A. Ward Ford Foundation (BC); Contract grant sponsor: National Institutes of Health; Contract grant numbers: AR51443, AR47551, AR248458, EB002495; Contract grant sponsor: National Institutes of Health Laser Microbeam and Medical Program; Contract grant number: P41-RR01192.

\section{References}

1. Tallman B, Tan OT, Morelli JG, Piepenbrink J, Stafford TJ, Trainor S, Weston WL. Location of portwine stains and the likelihood of ophthalmic and or central-nervous-system complications. Pediatrics 1991;87(3):323-327. [PubMed: 1805804] 
2. Nelson JS, Geronemus RG. Redarkening of port-wine stains 10 years after laser treatment. N Engl J Med 2007;356(26):2745-2746. [PubMed: 17596612]

3. van der Horst CMAM, Koster PHL, de Borgie CAJM, Bossuyt PMM, van Gemert MJC. Effect of the timing of treatment of port-wine stains with the flash-lamp-pumped pulsed dye-laser. N Engl J Med 1998;338(15):1028-1033. [PubMed: 9535667]

4. Lanigan SW. Port-wine stains unresponsive to pulsed dye laser: Explanations and solutions. $\mathrm{Br} \mathbf{J}$ Dermatol 1998;139(2):173-177. [PubMed: 9767228]

5. Randeberg LL, Bonesronning JH, Dalaker M, Nelson JS, Svaasand LO. Methemoglobin formation during laser induced photothermolysis of vascular skin lesions. Lasers Surg Med 2004;34(5):414-419. [PubMed: 15216535]

6. Troilius A, Wardell K, Bornmyr S, Nilsson GE, Ljunggren B. Evaluation of port wine stain perfusion by laser Doppler imaging and thermography before and after argon-laser treatment. Acta Derm Venereol 1992;72(1):6-10. [PubMed: 1350150]

7. Jernbeck J, Malm M. Calcitonin gene related peptide increases the blood-flow of port-wine stains and improves continuous-wave dye-laser treatment. Plast Reconstr Surg 1993;91(2):245-251. [PubMed: 8430139]

8. McGill DJ, Mackay IR. The effect of ambient temperature on capillary vascular malformations. Br J Dermatol 2006;154(5):896-903. [PubMed: 16634893]

9. Zhao YH, Chen ZP, Saxer C, Shen QM, Xiang SH, de Boer JF, Nelson JS. Doppler standard deviation imaging for clinical monitoring of in vivo human skin blood flow. Opt Lett 2000;25(18):1358-1360. [PubMed: 18066216]

10. Nelson JS, Kelly KM, Zhao YH, Chen ZP. Imaging blood flow in human port-wine stain in situ and in real time using optical Doppler tomography. Arch Dermatol 2001;137(6):741-744. [PubMed: 11405763]

11. Ren HW, Ding ZH, Zhao YH, Miao JJ, Nelson JS, Chen ZP. Phase-resolved functional optical coherence tomography: Simultaneous imaging of in situ tissue structure, blood flow velocity, standard deviation, birefringence, and Stokes vectors in human skin. Opt Lett 2002;27(19):17021704. [PubMed: 18033341]

12. Huang YC, Ringold TL, Nelson JS, Choi B. Noninvasive blood flow imaging for real-time feedback during laser therapy of port wine stain birthmarks. Lasers Surg Med 2008;40(3):167-173. [PubMed: 18366081]

13. Fercher AF, Briers JD. Flow visualization by means of single-exposure speckle photography. Opt Commun 1981;37(5):326-330.

14. Choi B, Kang NM, Nelson JS. Laser speckle imaging for monitoring blood flow dynamics in the in vivo rodent dorsal skin fold model. Microvasc Res 2004;68(2):143-146. [PubMed: 15313124]

15. Choi B, Ramirez-San-Juan JC, Lotfi J, Nelson JS. Linear response range characterization and in vivo application of laser speckle imaging of blood flow dynamics. J Biomed Opt 2006;11(4):041129. [PubMed: 16965157]

16. Choi B, Jia WC, Channual J, Kelly KM, Lotfi J. The importance of long-term monitoring to evaluate the microvascular response to light-based therapies. J Invest Dermatol 2008;128(2):485-488. [PubMed: 17657245]

17. Ramirez-San-Juan JC, Ramos-Garcia R, Guizar-Iturbide I, Martinez-Niconoff G, Choi B. Impact of velocity distribution assumption on simplified laser speckle imaging equation. Opt Express 2008;16 (5):3197-3203. [PubMed: 18542407]

18. Haedersdal M, Bech-Thomsen N, Therkildsen P, Poulsen T, Wulf HC. Impact of epidermal thickness on purpura from the pulsed dye laser. Lasers Surg Med 1998;22(3):159-164. [PubMed: 9510100]

19. Kelly KM, Choi B, McFarlane S, Motosue A, Jung BJ, Khan MH, Ramirez-San-Juan JC, Nelson JS. Description and analysis of treatments for port-wine stain birthmarks. Arch Facial Plast Surg 2005;7 (5):287-294. [PubMed: 16172335]

20. Choi B, Majaron B, Nelson JS. Computational model to evaluate port wine stain depth profiling using pulsed photothermal radiometry. J Biomed Opt 2004;9(2):299-307. [PubMed: 15065895]

21. Svaasand LO, Fiskerstrand EJ, Kopstad G, Norvang LT, Svaasand EK, Nelson JS, Berns MW. Therapeutic response during pulsed laser treatment of port-wine stains: Dependence on vessel diameter and depth in dermis. Laser Med Sci 1995;10(4):235-243. 
22. Selim MM, Kelly KM, Nelson JS, Wendelschafer-Crabb G, Kennedy WR, Zelickson BD. Confocal microscopy study of nerves and blood vessels in untreated and treated port wine stains: Preliminary observations. Dermatol Surg 2004;30(6):892-897. [PubMed: 15171768]

23. Bernstein EF. Treatment of a resistant port-wine stain with a new variable pulse-duration pulsed-dye laser. J Cosmet Dermatol 2008;7(2):139-142. [PubMed: 18482019]

24. Hohenleutner U, Hilbert M, Wlotzke U, Landthaler M. Epidermal damage and limited coagulation depth with the flashlamp-pumped pulsed dye-laser-A Histochemical-Study. J Invest Dermatol 1995;104(5):798-802. [PubMed: 7738359]

\section{APPENDIX}

\section{TABLE A1}

Treatment Parameters Used on Each Subject Enrolled in the Study

\section{SubjectGenderAgeLocations of PWS}

1 F 18 Bilateral facial

F $\quad 47$ Extensive right facial and neck
Laser wavelength (nm) Spot Size (mm)

Laser dosage $\left(\mathrm{J} / \mathrm{cm}^{2}\right)$

Pulse duration (ms)

Cryogen cooling spurt, delay)

Vbeam $(10)$
Vbeam $(9,10)$

Vbeam $(9,10)$

Vbeam (10)

Vbeam (595)

Cynergy $(595,1,064)$ and Cynergy $(10)$,

Vbeam (595) (10)

Alexandrite (755), VbeamAlexandrite (10),

(595)

Vbeam $(12,10)$

Cynergy $(8,30)$, Vbeam $(10)$

Alexandrite (40), Vbeam (6.5-10)

Vbeam (595), Ruby (694) Vbeam (10), Ruby (5) Vbeam (6 -10), Ruby (4.5)

(3)

Cynergy $(595,1,064)$ and Cynergy $(10)$, Vbeam Cynergy $(9,30)$, Vbeam $(6-C y$

Vbeam (595) (12) 9)

Vbeam (595), then CbeamVbeam (7), Cbeam (10)Vbeam (12), Cbeam (6)

(585)

Vbeam (595), then CbeamVbeam (10), Cbeam Vbeam (10), Cbeam (5)

(585)

(10)

3

F 51 Extensive left facial

Cynergy $(595,1,064)$

Cyner

Cbeam (585)

20 Extensive left facial

Vbeam (595)

$5 \quad \mathrm{~F} 25$ Exten

$\begin{array}{lll}6 & \text { M } & \text { 16 Left face, ear, neck } \\ 7 & \text { M } & \text { 23 Extensive left facial }\end{array}$

Vbeam $(595)$

Cbeam (585) Cbeam (8)

Vbeam (7)

Vbeam (8)

Vbeam $(10)$

Vbeam $(6.5-8.75)$ (10)

Cynergy $(595,1,064)$ thenCyn

Vbeam (595) (10)

F 18 Extensive bilateral facialVbeam (595)

Alexandrite (755), then

Cbeam (585)

Alexandrite (755), then

Vbeam (595)

9

F 18 Extensive PWS entire Vbeam (595) face, neck

Cynergy (595, 1,064)

Vbeam (595)

Alexandrite (755)

10

$\mathrm{M}$

52 Extensive bilateral facialCynergy (595) then and neck

Cbeam (585)

11 M 14 Extensive left facial Vbeam (595)

12 M 35 Extensive left facial withCynergy $(595,1,064)$ Sturge Weber

$\begin{array}{llll}13 & \text { F } & 19 \text { Extensive left facial } & \begin{array}{l}\text { Coeam (585) } \\ \text { Modified Vbeam (577) }\end{array} \\ 14 & \text { M } & 10 \text { Extensive right facial } & \text { Vbeam (595) } \\ & & & \\ 15 & \text { M } & \text { 37 Extensive right facial } & \text { Alexandrite (755) } \\ 16 & \text { M } & \text { 65 Extensive left facial } & \text { Cbeam }(585) \\ 17 & \text { M } & \text { 39 Chin and lip } & \text { Vbeam }(595)\end{array}$

Alexandrite (755), then (10)

Vbeam (12)

Alexandrite (10), then Alexandrite (60), then

Cbeam (10)

Alexandrite (10), then Alexandrite (40), then

Vbeam (12)

Vbeam (12)

Cynergy (10)

Vbeam (12)

Alexandrite (10)

Cynergy (10) then

Cbeam (10)

Vbeam (7)

Cynergy (10)

Alexandrite (10), then Cbeam (10)

Modified Vbeam (7)

Vbeam (595)

Alexandrite (10)

Cbeam (10)

Vbeam (10)
(0.45)

Vbeam $(1.5,10,20) \quad$ Vbeam $(30,30)$

Vbeam $(0.45,3,10) \quad$ Vbeam $(30,30)$

Vbeam $(0.45) \quad$ Vbeam $(30,30)$

Cynergy $(2,15)$, VbeamForced chilled air $(6,10)$

Alexandrite (3), VbeamAlexeandrite (50,

$(0.45,3,6) \quad 30)$, Vbeam $(40,20)$ (NA)
Vbeam (30, 30)
Ruby (NA) Vbeam (0.45), Cbeam Vbeam $(30,30)$,

Vbeam $(3,6)$, Cbeam Vbeam $(40,40)$,

(0.45) Cbeam $(40,20)$

Cynergy $(2,15)$

Cbeam (0.45) Cbeam $(30,30) \quad 40$

Vbeam $(0.45) \quad$ Vbeam $(30,30) \quad 400$

Vbeam $(0.45,3,10) \quad$ Vbeam $(30,50) \quad 40$ Cynergy $(2,15)$, CbeamCynergy(chilled air), 350

$\begin{array}{cc}(0.45) & \text { Cbeam }(30,30) \\ \text { Cynergy }(8,30) \text {, Vbeam (10)Cynergy }(2,15) \text {, VbeamCynergy (chilled } 350\end{array}$

Cynergy $(2,15)$, VbeamCynergy (chilled
$(3,6,10)$
air), Cbeam $(30,30)$ Vbeam $(0.45,10) \quad$ Vbeam $(30,30) \quad 900$ Alexandrite (3), CbeamAlexandrite $(50,30), 900$ (0.45) Cbeam $(30,30)$

Alexandrite (3), VbeamAlexandrite $(50,30), 300$

$\begin{array}{lll}\text { Vbeam }(6.5-10) & (0.45,3,6) & \text { Vbeam }(40,20) \\ \text { Vbeam }(6-7) & \text { Vbeam }(0.45,3,10) & \text { Vbeam }(30,30)\end{array}$

Cynergy $(9,30)$

Vbeam (6.5)

Cynergy (40)

Alexandrite (70)

Vbeam (0.45)

Cynergy (10) then Cbeam (8)Cynergy (6), Cbeam Cynergy (chilled 1,200

Vbeam (10)

$(0.45)$

Vbeam $(3,20)$

air), Cbeam $(30,30)$

Cynergy $(8,25-35)$

Cynergy $(2,15)$

Alexandrite (3), then Alexandrite (50,30), 220

Cbeam

Cbeam $(30,30)$

Cbeam (6)

Modified Vbeam (0.45)Modified Vbeam $(30,30)$

Vbeam $(20,10,6,3,1.5$,Vbeam $(30,30)$

$0.45)$

Alexandrite (3)

Cbeam (0.45)

Alexandrite (60)

Cbeam (7)

Vbeam (20)

Vbeam $(30,30)$ 600

Cynergy (chilled air) 90

1,700

Cynergy (chilled air) 1,000

Vbeam $(30,30) \quad 1,200$

beam $(30,30) \quad 25$

Alexandrite $(50,30) \quad 150$

Cbeam $(40,20) \quad 300$ 


\begin{tabular}{|c|c|c|c|c|c|c|c|c|}
\hline \multicolumn{3}{|c|}{ SubjectGenderAgeLocations of PWS } & \multirow{3}{*}{$\begin{array}{l}\text { Laser wavelength (nm) } \\
\text { Cbeam (585), Vbeam } \\
(595) \\
\text { Vbeam (595) }\end{array}$} & \multirow{2}{*}{$\begin{array}{l}\text { Spot Size }(\mathbf{m m}) \\
\text { Cbeam (7), Vbeam (7) }\end{array}$} & \multirow{2}{*}{$\begin{array}{l}\text { Laser dosage }\left(\mathbf{J} / \mathbf{c m}^{2}\right) \\
\text { Cbeam (5.5), Vbeam (7) }\end{array}$} & \multirow{3}{*}{$\begin{array}{l}\text { Pulse duration (ms) } \\
\text { Cbeam }(1.5,6) \text {, Vbeam } \\
\text { (3) } \\
\text { Vbeam (3) }\end{array}$} & \multirow{2}{*}{$\begin{array}{c}\begin{array}{l}\text { Cryogen cooling } \\
\text { (spurt, delay) }\end{array} \\
\text { Cbeam }(30,30), \\
\text { Vbeam }(30,30)\end{array}$} & \multirow{2}{*}{$\begin{array}{c}\begin{array}{c}\text { Entire } \\
\text { treated } \\
\text { area } \\
\left(\mathbf{c m}^{2}\right)\end{array} \\
30\end{array}$} \\
\hline & & & & & & & & \\
\hline & & & & Vbeam (7) & $\begin{array}{l}\text { Left-Vbeam (7), right- } \\
\text { Vbeam (7.5) }\end{array}$ & & Vbeam $(30,30)$ & 30 \\
\hline 18 & $\mathrm{~F}$ & 18 Left leg & $\begin{array}{l}\text { Alexandrite (755), then } \\
\text { Vbeam (595) }\end{array}$ & $\begin{array}{l}\text { Alexandrite (10), then } \\
\text { Vbeam (10) }\end{array}$ & $\begin{array}{l}\text { Alexandrite (80), then } \\
\text { Vbeam (10) }\end{array}$ & $\begin{array}{l}\text { Alexandrite (3), Vbean } \\
\text { (3) }\end{array}$ & $\begin{array}{c}\text { nAlexandrite }(50,30) \text {, } \\
\text { Vbeam }(30,30)\end{array}$ & 100 \\
\hline 19 & $\mathrm{~F}$ & $\begin{array}{l}45 \text { Extensive left facial, } \\
\text { neck, and chest }\end{array}$ & Vbeam (595) & Vbeam (12) & Vbeam $(6.5)$ & Vbeam $(0.45)$ & Vbeam $(40,20)$ & 750 \\
\hline 20 & F & 14 Extensive right facial & $\begin{array}{l}\text { Alexandrite ( } 755) \text {, then } \\
\text { Vbeam }(595) \text {, then } \\
\text { modified Vbeam ( } 577)\end{array}$ & $\begin{array}{l}\text { Alexandrite (12), then } \\
\text { Vbeam (10), modified } \\
\text { Vbeam ( } 7)\end{array}$ & $\begin{array}{l}\text { Alexandrite (40), Vbeam } \\
\text { (10), modified Vbeam (6.5) }\end{array}$ & $\begin{array}{l}\text { Alexandrite (3), Vbean } \\
\text { (3), modified Vbeam } \\
(0.45)\end{array}$ & $\begin{array}{l}\text { nAlexandrite }(50,30) \text {, } \\
\text { Vbeam }(40,20), \\
\text { modified Vbeam }(40, \\
20)\end{array}$ & 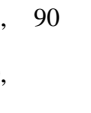 \\
\hline
\end{tabular}



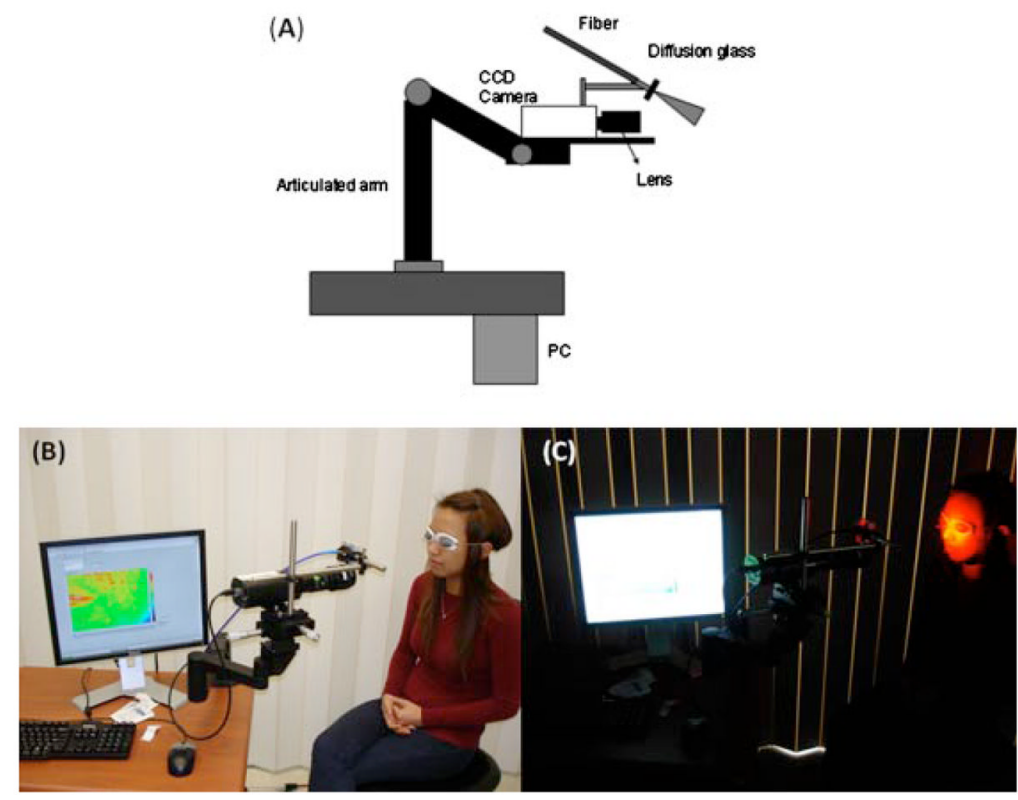

Fig. 1.

A: Schematic of clinical LSI instrument, first described in Ref. [12]. B, C: Clinical LSI instrument in action. 


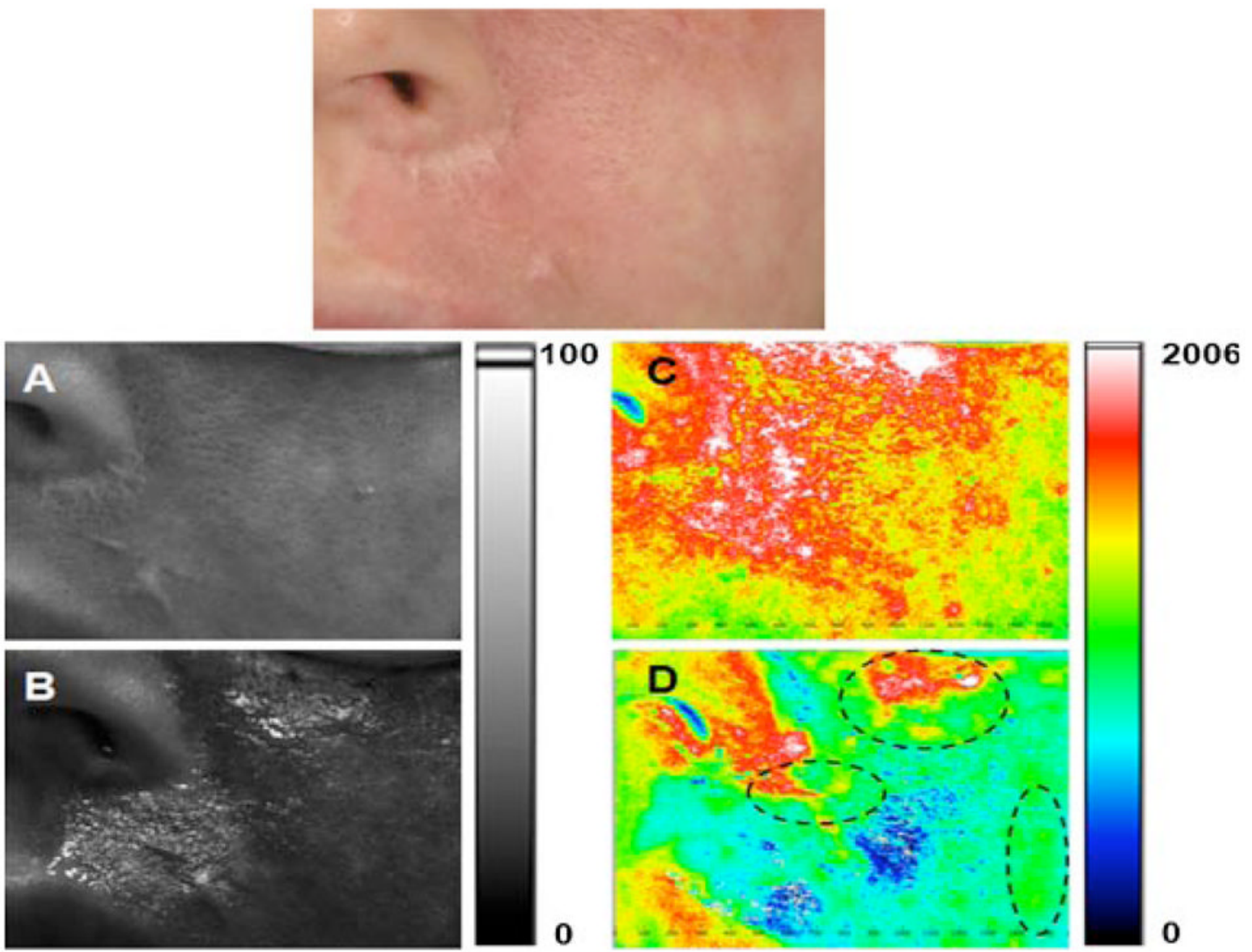

Fig. 2.

Persistent perfusion after laser therapy. Top: Photograph of user-specified region of interest (ROI) identified on a female Caucasian patient with a facial PWS birthmark. A, B: Grayscale and $(\mathbf{C}, \mathbf{D})$ corresponding SFI maps of ROI treated with a PDL. Images were acquired (A, C) immediately before, and (B, D) 40 minutes after, treatment. With the exception of the nose and lips, the entire region shown in A was treated. Purpura formation was evident throughout the treated region. The regions enclosed in dashed lines (D) correspond to regions of persistent perfusion, despite the formation of purpura. 


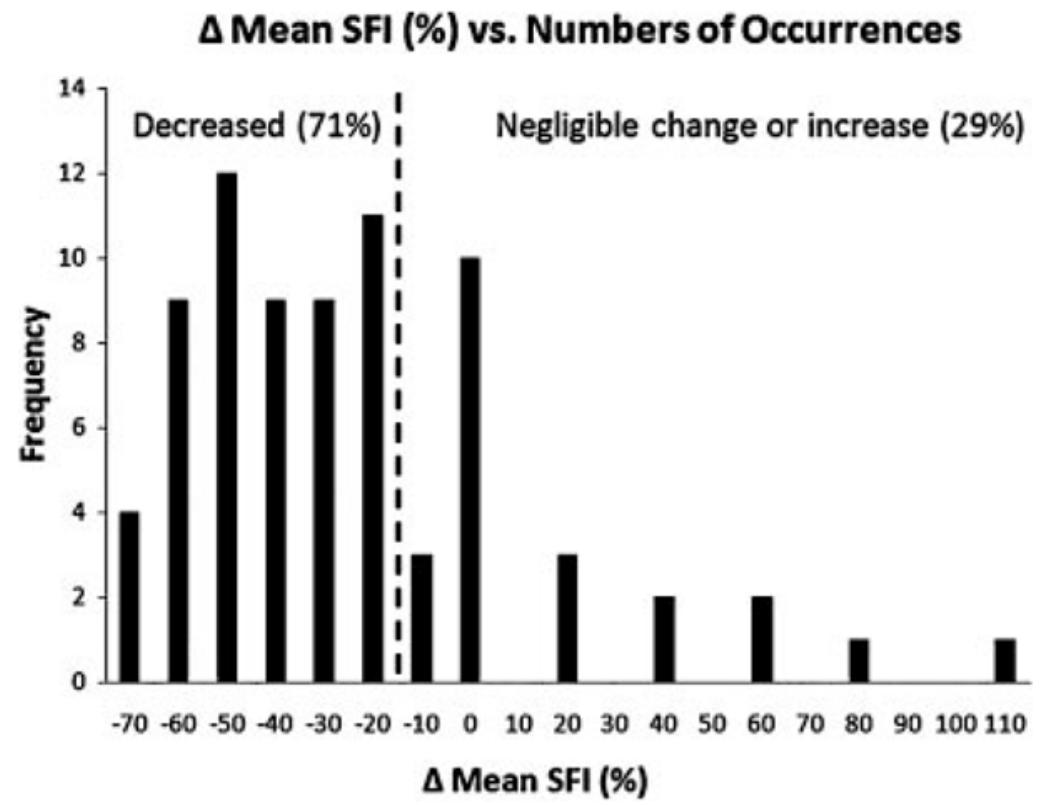

Fig. 3.

Based on SFI images collected before and 40 minutes after laser treatment, over 76 treatment sessions, we postulate that the acute vascular response to laser therapy varies considerably. A perfusion reduction $>20 \%$ (i.e., left of the dashed line) was observed in $71 \%$ of the treatment sessions. In contrast, in $12 \%$ of the treatment sessions, an increase in mean SFI was observed. 


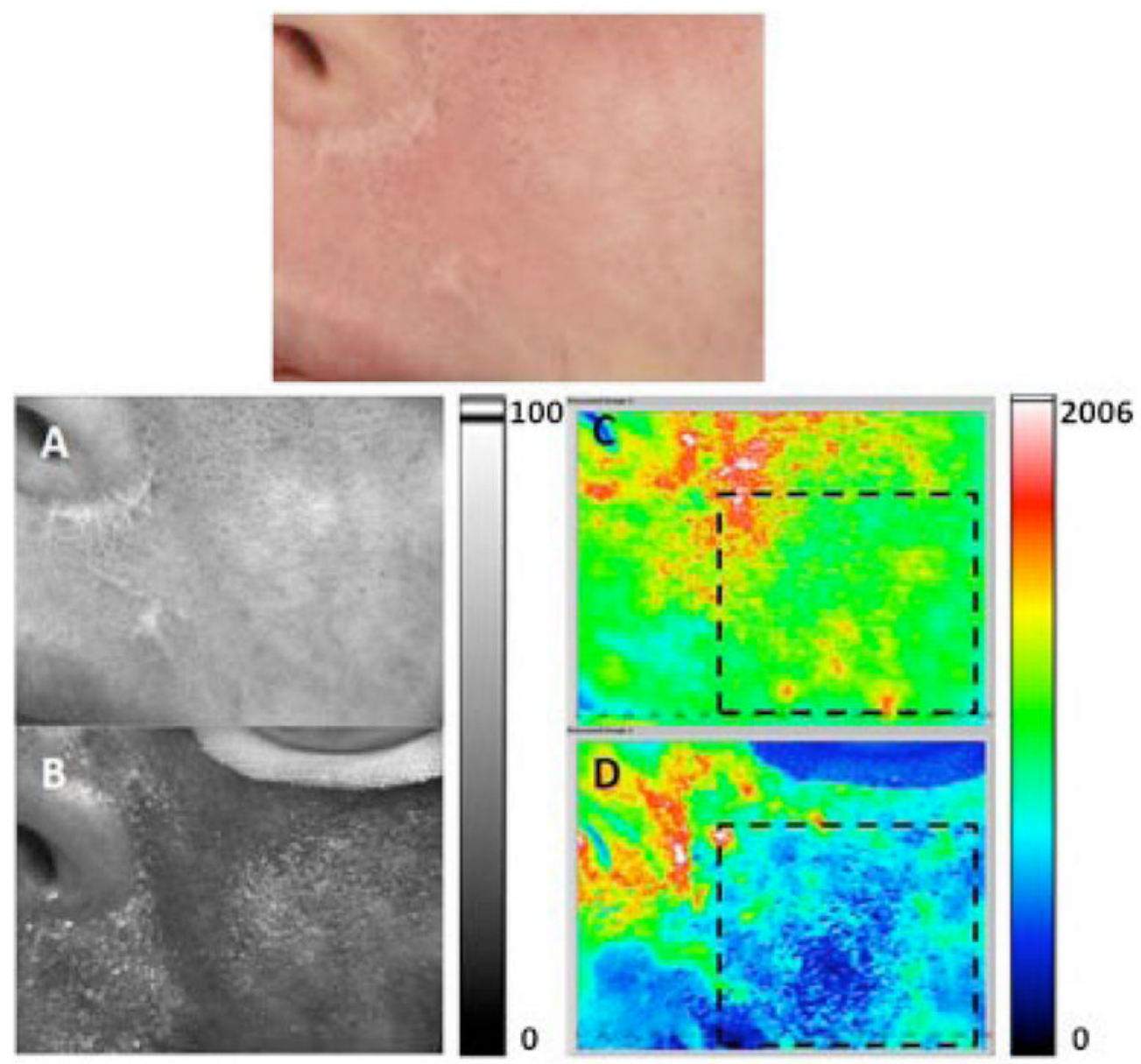

Fig. 4.

Example of moderate DoH achieved after laser therapy. Top: Photograph of user-specified region of interest (ROI) identified on a female Caucasian patient with a facial PWS birthmark. A, B: Grayscale and $(\mathbf{C}, \mathbf{D})$ corresponding SFI maps of ROI treated with a PDL. Images were acquired (A, C) immediately before, and (B, D) 40 minutes after, treatment. Areas enclosed in dashed lines in (C) and (D) were selected to calculate the change in perfusion and DoH. B perfusion was decreased by $50 \%$ after treatment, with a post-treatment DoH of 0.38 (i.e., moderate level). 


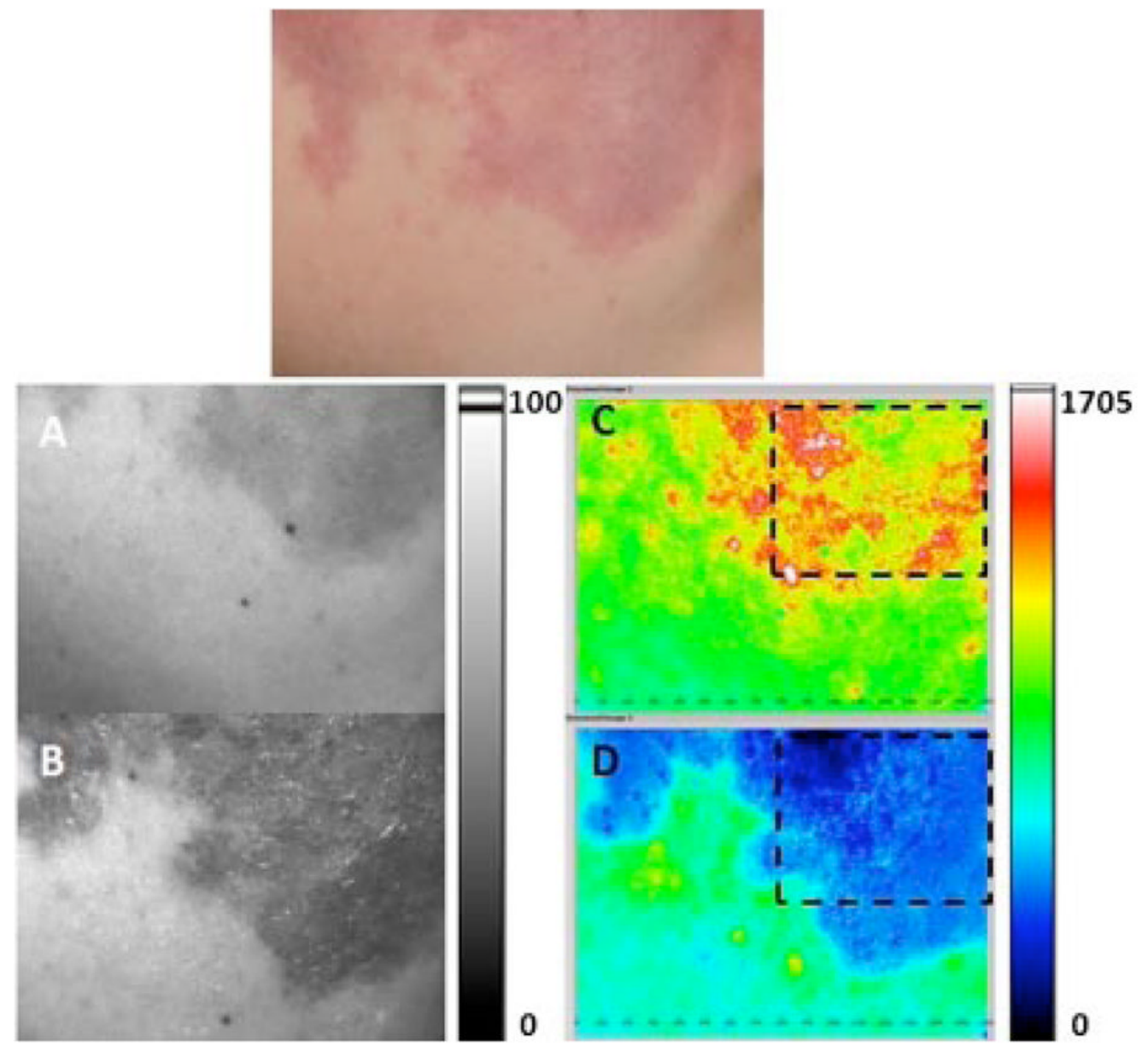

Fig. 5.

Example of low DoH achieved after laser therapy. Top: Photograph of user-specified region of interest (ROI) identified on a female Caucasian patient with a facial PWS birthmark. A, B: Grayscale and (C, D) corresponding SFI maps of ROI treated with a pulsed dye laser. Images were acquired (A, C) immediately before, and (B, D) 40 minutes after, treatment. Areas enclosed in dashed lines in (C) and (D) were selected to calculate the change in perfusion and DoH. Blood perfusion decreased by $71 \%$ after treatment, with a post-treatment DoH of 0.27 (i.e., low level). 


\section{Degree of Heterogeneity}

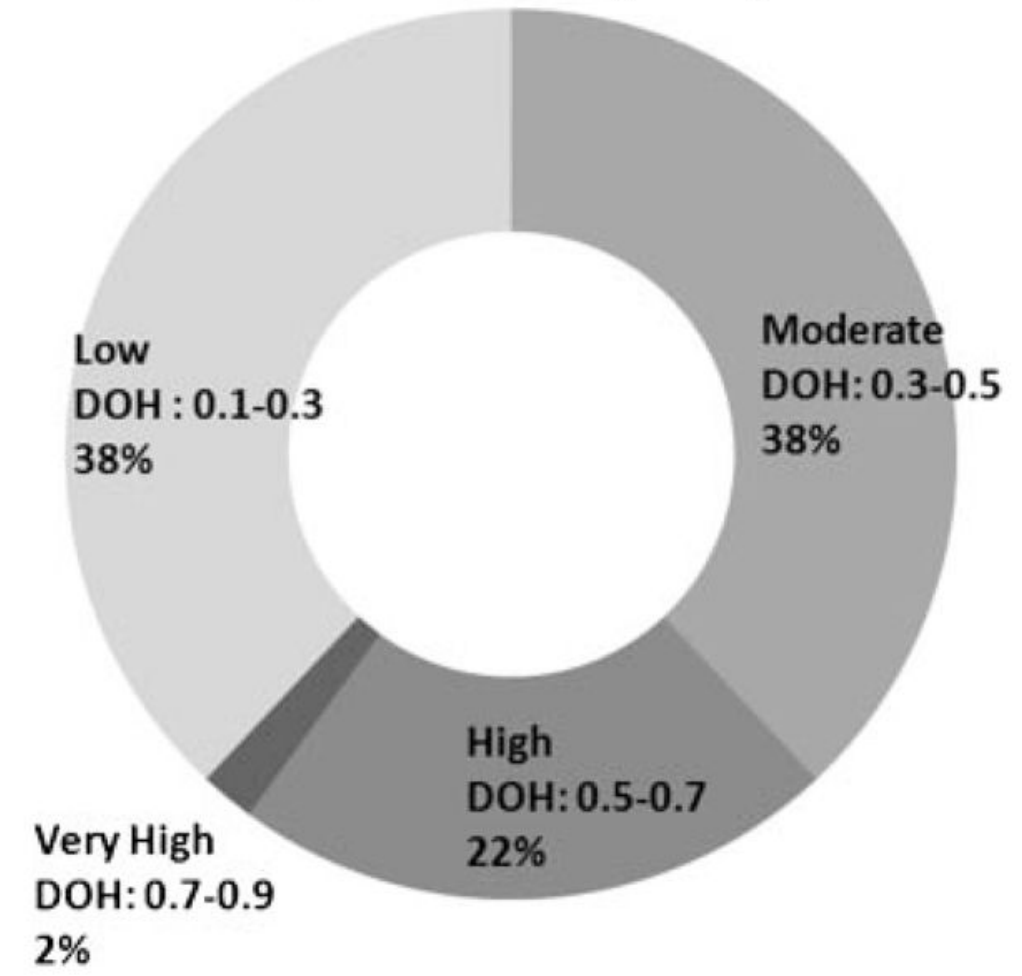

Fig. 6.

Summary of DoH outcomes for the 54 treatment sessions in which a mean perfusion of reduction of at least $20 \%$ was obtained. 


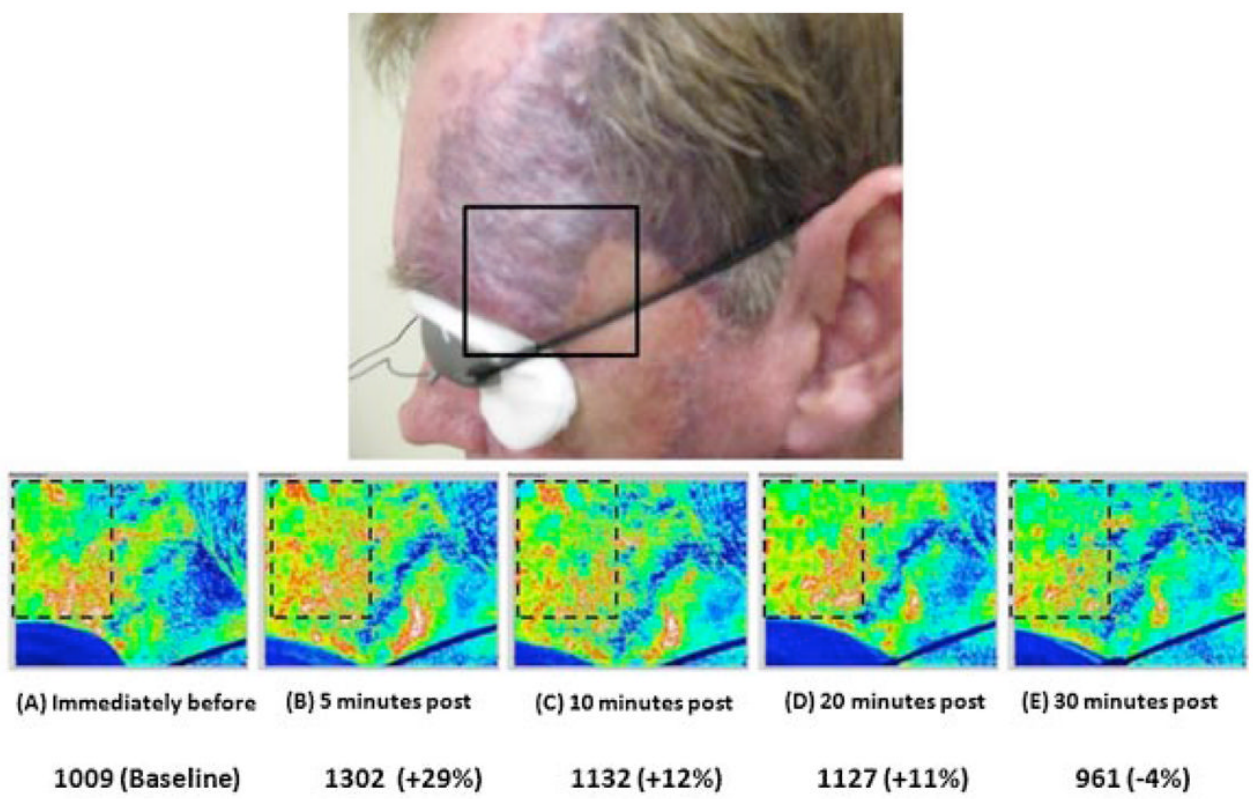

Fig. 7.

Over the initial 30 minutes after laser therapy, the perfusion of the treated PWS region is dynamic. Top: Photograph from male Caucasian patient with PWS on the left side of the face. Bottom: SFI images taken from ROI enclosed in the solid black line (A) immediately before; and (B) 5 minutes, (C) 10 minutes, (D) 20 minutes, and (E) 30 minutes post-treatment.

Compared to the baseline image (A), the mean SFI value of the region enclosed by the dashed line was $29 \%, 12 \%$, and $11 \%$ greater than baseline values at 5, 10, and 20 minutes after treatment, respectively. At 30 minutes after treatment, the mean SFI value was $4 \%$ below the baseline value. 